\title{
Ultrasonic focusing through the ribs using the DORT method
}

\author{
E. Cochard, ${ }^{\text {a) }}$ C. Prada, J. F. Aubry, and M. Fink \\ Laboratoire Ondes et Acoustique, CNRS UMR 7587, Université Paris VII, Inserm, ESPCI, 10 rue \\ Vauquelin, 75005 Paris, France
}

(Received 2 February 2009; revised 18 May 2009; accepted for publication 5 June 2009; published 1 July 2009)

\begin{abstract}
Thermal ablation induced by high intensity focused ultrasound has produced promising clinical results to treat hepatocarcinoma and other liver tumors. However skin burns have been reported due to the high absorption of ultrasonic energy by the ribs. This study proposes a method to produce an acoustic field focusing on a chosen target while sparing the ribs using the decomposition of the time-reversal operator (DORT method). The idea is to apply an excitation weight vector to the transducers array which is orthogonal to the subspace of emissions focusing on the ribs. A linear array of transducers has been used to measure the set of singular vectors associated with a chest phantom, made of three human ribs immersed in water, and to produce the desired acoustic fields. The resulting propagating fields have been measured both in the focal plane and in the plane of the ribs using a needle hydrophone. The ratio of the energies absorbed at the focal point and on the ribs has been enhanced up to 100-fold, as demonstrated by the measured specific absorption rates. (C) 2009 American Association of Physicists in Medicine. [DOI: 10.1118/1.3159755]
\end{abstract}

Key words: high intensity focused ultrasound, noninvasive surgery, DORT, phased array, adaptive focusing

\section{INTRODUCTION}

High intensity focused ultrasounds (HIFUs) allow noninvasive energy concentration onto soft tissue and can induce localized temperature elevation $\left(30-55^{\circ} \mathrm{C}\right)$ within a few seconds. The resulting high temperature can generate irreversible tissue necrosis while leaving the surrounding normal tissue undamaged. This method is used to treat a variety of conditions, such as benign prostate hyperplasia, ${ }^{1}$ prostate cancer, ${ }^{2-5}$ uterine fibroid, ${ }^{6}$ kidney tumors, ${ }^{7}$ and liver tumors. ${ }^{8}$

However, in the case of the liver, the presence of the rib cage is a major problem. It acts as an aberrator that affects the focusing ${ }^{9}$ and, due to the high absorption coefficient of the bones ${ }^{10}$ overheating of the ribs can be quite important. Using magnetic resonance temperature monitoring of pigs in vivo, Daum et al. reported temperature elevations during sonication five times higher on the ribs than in the intercostal space. ${ }^{11}$ This overheating can cause up to third-degree skin burns $^{8,12,13}$ and in some cases this has led to the surgical removal of part of the ribs. ${ }^{14}$

Several methods have been proposed to avoid the removal of ribs, all of which use a transducer array to produce a field that avoids sending energy on to the ribs. Botros et al. ${ }^{15}$ proposed a two-step method based on the introduction of a virtual array along the intercostal spacings. Two propagation matrices are defined first, one between the target points and the virtual array and the other between the virtual array and the focusing array. Thanks to the pseudoinversion of the first matrix, the particle velocities on the virtual array are computed first, so that the resulting field focuses on the desired location. Finally, the particle velocities on the actual array are computed so that the resulting field is the correct field on the virtual array. This numerical study demonstrated that it is possible to reduce the power on the ribs to $1 \%-3 \%$ of the total power. However, it requires the pseudoinversion of two propagation matrices and has not been tested on real ribs.

Another approach simply consists of switching off the array elements whose normal vectors cross the ribs (i.e., the elements in front of the ribs). Liu et al. showed, through a numerical simulation, that this reduces the temperature elevation at the ribs. ${ }^{16}$ This method could be very simple to implement but may not be the optimal method since it does not take into account wave diffraction by the ribs or shear mode conversion.

Time-reversal correction was first introduced to correct phase aberrations. As this technique is a matched filter, it maximizes the ratio between the energy deposition at focus and the total emitted energy in the nonabsorbing media as well as in the absorbing media. ${ }^{17}$ It therefore minimizes the energy deposition on the ribs. Aubry et al. showed experimentally that a mean decrease of $83 \%$ of the temperature elevation on the ribs could be achieved by time reversal compared to a noncorrected spherical wave front. ${ }^{17}$ However, this robust and real-time adaptive focusing method requires a transducer being placed at the desired focal point, making this technique invasive.

The present study proposes a novel noninvasive solution. The DORT method (French acronym for décomposition de l'opérateur de retournement temporel) was derived from the analysis of acoustic time-reversal mirrors. ${ }^{18}$ It consists of finding the invariants of the time-reversal process using the backscattered echoes recorded on the transmit/receive array. These invariants contain information about the medium and are used to avoid sending energy on the reflectors (in the present case, the ribs). Basically, the desired emission signals (i.e., focusing on the region to treat) are projected orthogonally to the set of emissions that focus on the ribs. This set 
can be determined by applying the DORT method to the ribs' echoes. As will be shown, this process allows the construction of an emission that focuses on the chosen point while minimizing the energy deposition on the ribs. A similar technique has been used by Song et al. in underwater acoustics, where an oceanic waveguide was insonified while minimizing reverberations from the bottom of the guide. ${ }^{19}$

In this study, a 128-element linear phased array and a chest phantom made of three ribs immersed in water were used to demonstrate the efficiency of the method. The specific absorption rate (SAR) was chosen as the indicator of efficiency. The SARs obtained after projection have been compared to those obtained without projection and those obtained by using the time-reversal technique.

\section{MATERIAL AND METHODS}

\section{II.A. Experimental setup}

The experiment was achieved with a linear array made of 128 transducers of $1.5 \mathrm{MHz}$ central frequency with an array pitch of $0.55 \mathrm{~mm}$ (Vermon PA1.5V1002). The transducers were driven by fully programmable parallel processed electronic channels of $20 \mathrm{MHz}$ sampling rate (Corelec, France). The dynamic was $90 \mathrm{~dB}$ in reception and $48 \mathrm{~dB}$ in emission (maximum peak to peak amplitude: $200 \mathrm{mV}$ in reception and $15 \mathrm{~V}$ in emission). This system allowed the user to insonify the medium and record the echoes in a flexible manner.

The pressure field propagating from the transducers was measured by a $0.4 \mathrm{~mm}$ needle hydrophone (Onda HNP-0400 attached to a Onda AH-2020 preamplifier) fixed to a threeaxis motor with $10 \mu \mathrm{m}$ precision. The fields were recorded first in the focal plane and second in the plane of the ribs once the ribs had been removed.

Three human ribs were placed in a geometry mimicking a human rib cage. In accordance with the local ethics committee, the ribs were extracted from a person who gave his consent to donate his body for medical research. After extraction, the ribs were immersed in boiling water for several days, then in trichloroethylen, and finally dehydrated. After complete sterilization, they were lent to the Laboratoire Ondes et Acoustique for research. The ribs were then immersed in water and degassed for a week in a vacuum chamber at $60 \mathrm{~mm} \mathrm{Hg}$ so that no air bubbles stayed trapped in the porous zones. The purpose of the degazing was to reproduce in vivo conditions as closely as possible. The width of the ribs varied from 10 to $15 \mathrm{~mm}$ and their thickness from 5 to $10 \mathrm{~mm}$. They were attached to a frame and the intercostal space was varied from 12 to $17 \mathrm{~mm}$ depending on the experiment. Their distance to the echographic probe was $30 \pm 5 \mathrm{~mm}$. The location of the focal spot was chosen to be consistent with the location of an actual spot to treat. The distance between the chosen focal point and the echographic array was $77 \mathrm{~mm}$. It was also in front of the array and roughly centered (facing the 72nd transducer). The layouts of the array, the ribs, and the focal spot are displayed in Figs. 1(a) and 1(b).
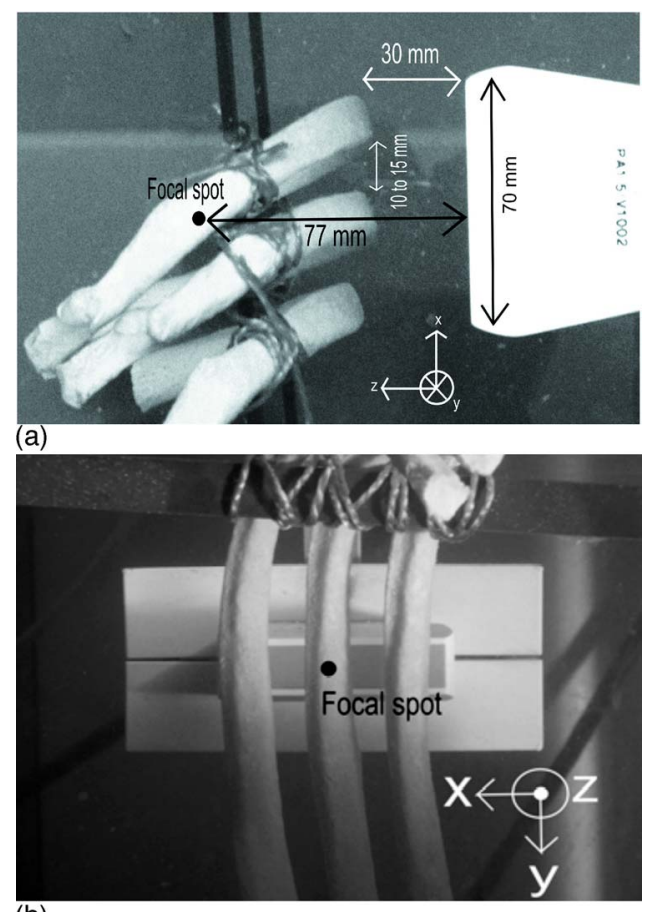

(b)

FIG. 1. (a) Annotated picture of the ribs viewed from the top. (b) Annotated picture of the ribs viewed from the side.

\section{II.B. Specific absorption rate}

To evaluate the efficiency of the method and compare the results from different sets of measurements, the SAR (in $\mathrm{W} \mathrm{m}^{-3}$ ) at a given position $x$ is defined as follows:

$$
\operatorname{SAR}(x)=\alpha(x) \frac{\left|P\left(x, f_{c}\right)\right|^{2}}{2 \rho(x) c(x)},
$$

where $\alpha(x)$ is the absorption coefficient at position $x, P$ is the Fourier component of the pressure at point $x$ (so that $P^{2} / 2$ is the mean squared pressure for a monochromatic emission) and at the central frequency $f_{c}$, and $\rho c$ is the acoustic impedance. The SAR gain is defined as the ratio of the SAR on the target to the SAR on the point to spare. There is actually a whole area to spare. For this reason, we calculated the SAR on "the point to spare" in two different ways: As the spatial average of the SARs measured along the central rib surface (which was actually a line since we worked on a 2D geometry) and as the maximum SAR observed on the ribs. The first SAR gain estimates the efficiency of the method if thermal diffusion (which tends to homogenize the temperature) is sufficient, while the second SAR gain informs about the hot spot level (masked by the spatial averaging) and is relevant if diffusion is low. To relate the field distribution to temperature elevation would require further analysis which we believe is beyond the scope of this paper.

As the focal point was chosen behind the central rib, the average was not calculated over the lateral ribs that were less insonified. Taking this average would artificially increase the SAR gain for any focusing emission. A SAR gain of at least unity indicates a higher energy absorption rate at the targeted 


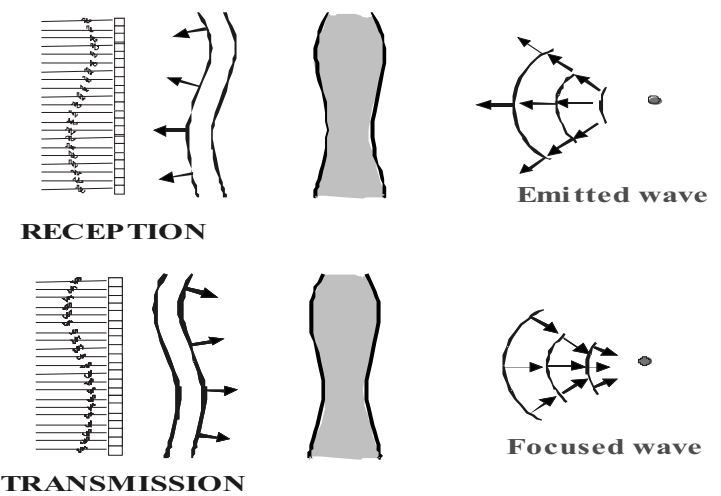

FIG. 2. Principle of time-reversal mirror.

focal spot than on the ribs. We chose to calculate the SAR for a single frequency because monochromatic signals are used for therapy (typically several second pulse length).

The values of $\alpha, \rho$, and $c$ are taken from Liu et al. ${ }^{16}$ except for the absorption coefficient of the bone, which is taken from Fry et al. ${ }^{20}$

(a) $\alpha_{\text {liver }}=\mu_{\text {focal }}=13.5 \mathrm{~Np} \mathrm{~m}^{-1}$ and $\alpha_{\text {bone }}=214 \mathrm{~Np} \mathrm{~m}^{-1}$, where $\alpha$ is the absorption coefficient and $\mu$ is the attenuation coefficient.

(b) $\rho_{\text {liver }}=1055 \mathrm{~kg} \mathrm{~m}^{-3}$ and $\rho_{\text {bone }}=1450 \mathrm{~kg} \mathrm{~m}^{-3}$.

(c) $\quad c_{\text {liver }}=1547 \mathrm{~m} \mathrm{~s}^{-1}$ and $c_{\text {bone }}=2300 \mathrm{~m} \mathrm{~s}^{-1}$.

The values at the focal spot correspond to the values of the liver.

\section{II.C. Time reversal}

Time reversal is a focusing method that relies on the invariance of the wave equation under time reversal. ${ }^{21}$ It requires an array of transducers and a source at the desired focal point. ${ }^{22}$

The wave propagating from an impulse point source to the array of transducers is recorded and time reversed. If the medium is time invariant, the time reversed signal propagates as if going backward in time and converges to the initial spot as illustrated in Fig. 2. This method is robust and efficient even in a complex propagating medium.

A scatterer embedded in the medium acts as a source by reflecting the energy it receives. By insonifying the medium and recording the backscattered wave, one can learn to focus on the scatterer. But in the presence of multiple scatterers, time reversal does not teach how to focus on each of them separately.

\section{II.D. Decomposition of the time-reversal operator}

The DORT method was derived from the theoretical study of iterative time-reversal mirrors. It consists essentially of the construction of the wave fronts that are invariable under a time-reversal process. ${ }^{23}$ These invariants appear as the eigenvectors of a matrix called the time-reversal operator, which describes the time-reversal process.

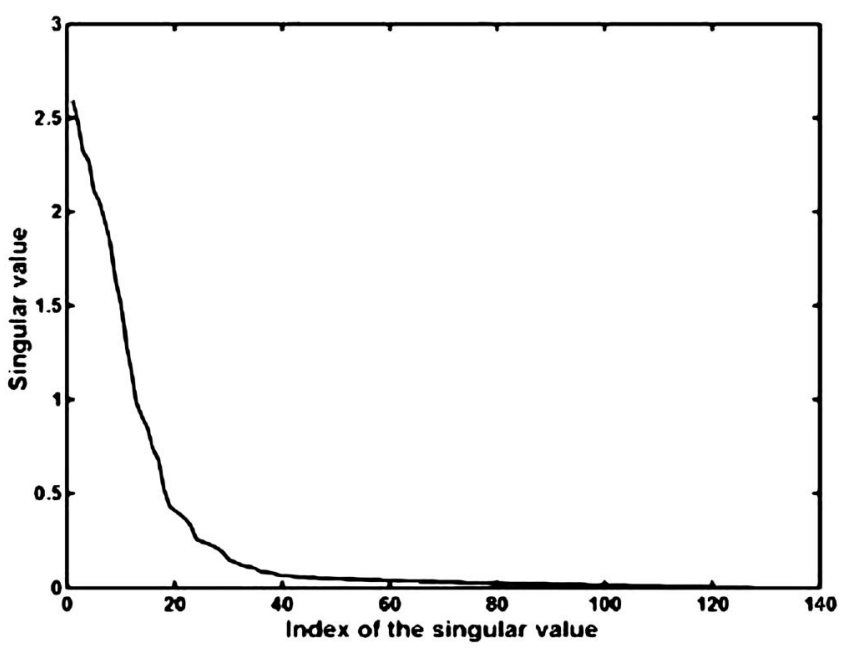

FIG. 3. Typical singular values distribution at the central frequency $(1.5 \mathrm{MHz})$.

Considering an array of $\mathrm{N}$ transducers and assuming that the system is linear and time invariant, one can build in the frequency domain a $N^{*} N$ matrix $\mathbf{K}(\omega)$, the elements of which are the interelement transfer functions at the pulsation $\omega$. The time reversal operator (TRO) is then defined as $\mathbf{K}(\omega) \mathbf{K}^{*}(\omega){ }^{23}$

This operator describes the iteration of the following sequence: Transmission, reception, and time-reversal operation (equivalent to a phase conjugation in the frequency domain). It is a Hermitian operator with positive eigenvalues. Its eigenvectors are the invariant wave forms of the timereversal process [if an eigenvector of the time-reversal operator $\mathbf{V}(\omega)$ is the transmitted signal, then the signal received is $\boldsymbol{\lambda}(\omega) \mathbf{V}(\omega)$.

From a mathematical point of view, the diagonalization of the TRO is equivalent to the singular value decomposition (SVD) of the array response matrix $\mathbf{K}(\omega)$. The SVD decomposition is $\boldsymbol{K}(\boldsymbol{\omega})=\boldsymbol{U}(\boldsymbol{\omega}) \boldsymbol{\Lambda}(\boldsymbol{\omega}) \boldsymbol{V}^{\dagger}(\boldsymbol{\omega})$, where $\boldsymbol{\Lambda}$ is a real diagonal matrix and $\boldsymbol{U}$ and $\boldsymbol{V}$ are unitary matrices. This means that the columns of $\boldsymbol{V}$ are the eigenvectors of the TRO and the singular values are the square roots of the eigenvalues. ${ }^{23}$

It is now well known that if the medium is composed of several pointlike, isotropic, and well resolved scatterers, the number of nonzero eigenvalues of the TRO is equal to the number of scatterers and each eigenvector is associated with one scatterer. ${ }^{23}$ This means that applying the phases and amplitudes of a chosen eigenvector to the elements of the array produces a wave front focusing on the corresponding scatterer. In general, a scatterer is not isotropic or pointlike and is associated with several invariants of the time-reversal operator. These invariants were studied by Aubry et al. in the case of a solid cylinder. ${ }^{24}$ The number of invariants is equal to the rank of $\mathbf{K}(\boldsymbol{\omega})$, which is on the order of the number of resolution cells in the scatterer. ${ }^{25}$ Each eigenvector associated with a significant eigenvalue focuses on one portion of the scatterer. 

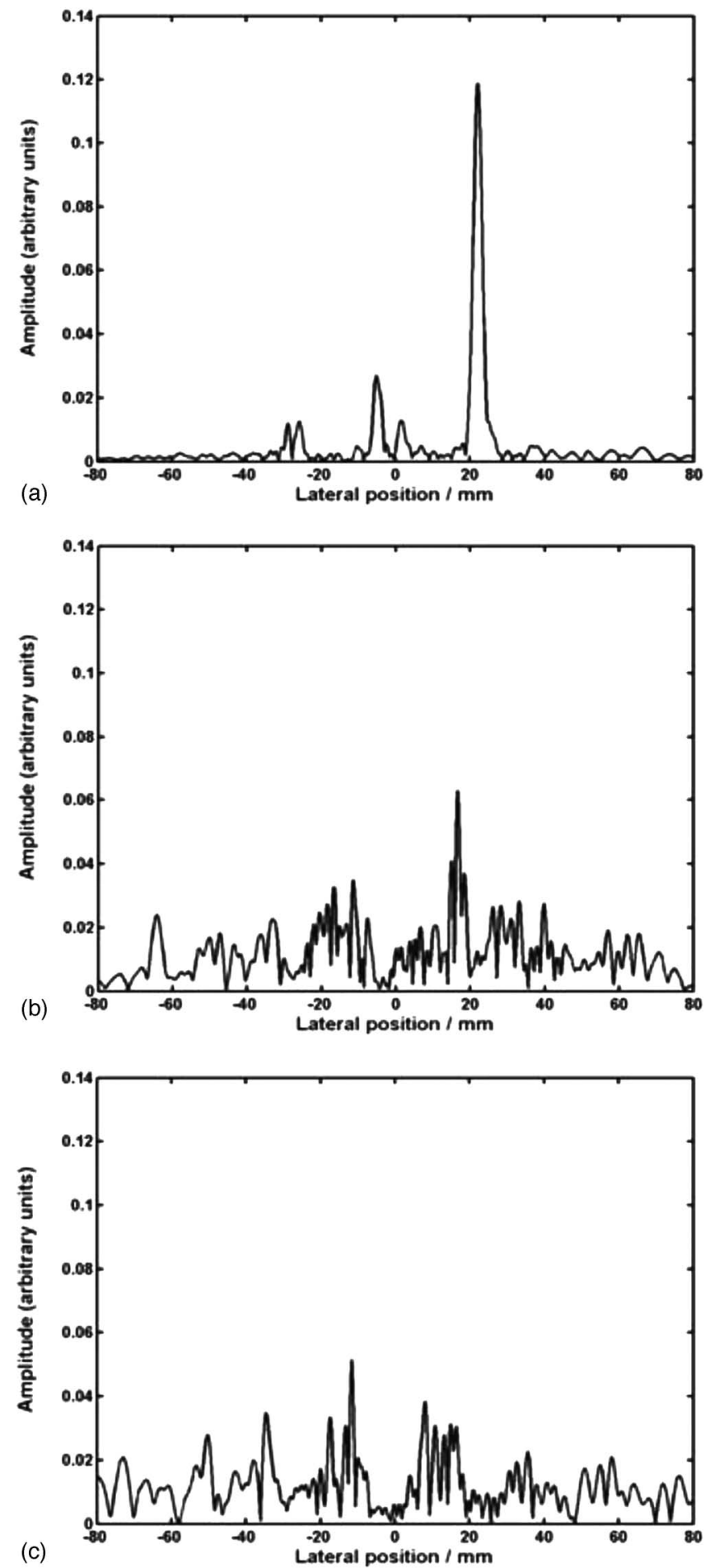

FIG. 4. Numerical repropagation of the eigenvectors from the echographic array to the plane of the ribs $(f=1.5 \mathrm{MHz})$. The lateral position at $0 \mathrm{~mm}$ corresponds to the center of the echographic array: (a) first eigenvector, (b) 37th eigenvector, and (c) 40th eigenvector.

In practice, the array response matrix $\mathbf{K}$ is measured by emitting a pulse on each array element successively and measuring the corresponding echoes on the $\mathrm{N}$ transducers. An appropriate time window is then used to select the echoes from the ribs, and a Fourier transform of the selected signals is performed at each frequency within the transducers band-

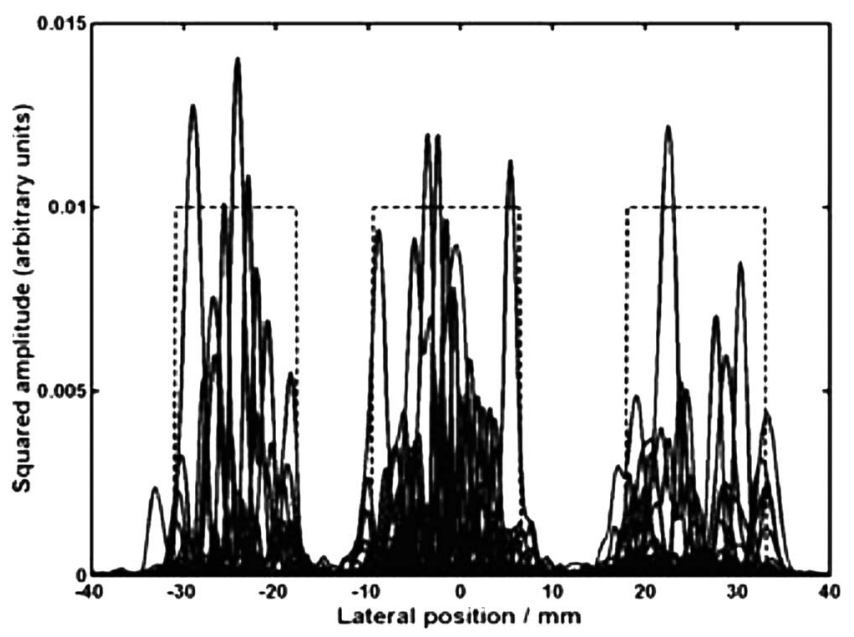

FIG. 5. Superposition of the numerical backpropagation of the first 20 eigenvectors from the echographic array to the plane of the ribs. The lateral position at $0 \mathrm{~mm}$ corresponds to the center of the echographic array. The ribs position is shown by a dotted line $(f=1.5 \mathrm{MHz})$.

width. Then, looking at the singular values, the singular vectors are separated into two categories: A first set focusing on the ribs (the eigenvectors associated with highest singular values) and a second set, the eigenvectors associated with the smallest singular values, that do not send energy on to the scatterers.

\section{II.E. Projection}

The first step of the method consists of defining a set of emission signals $\boldsymbol{A}(\omega)$ focusing on the target point, independent of the effect of the ribs. To this end, a time-reversal experiment was performed in the absence of the ribs: Each transducer emitted an impulse and the 128 resulting propagated fields were measured by an hydrophone located at the target location. Due to reciprocity, these A-scans form the B-scan that would be measured on the array if an impulse was emitted by the hydrophone. This B-scan is then time reversed. The Fourier transform of this time-reversed B-scan gives rise to the $\boldsymbol{A}(\omega)$ on demand. If this B-scan is used to focus through the ribs, part of the wave front is not affected by the ribs and focuses at the desired point but part of it is absorbed and scattered by the ribs. Instead of using a timereversal experiment, a conventional noncorrected cylindrical wave front could have been used. Nevertheless, due to time reversal, the phase defects of the transducers are automatically corrected. Moreover, such signals will have the same bandwidth as the interelements impulse response, thus enabling a fair comparison between all the techniques presented in this article. In the whole paper, the "noncorrected" signals will be $\mathbf{A}(\omega)$.

$\boldsymbol{A}(\omega)$ is then projected orthogonally to the first set of eigenvectors 


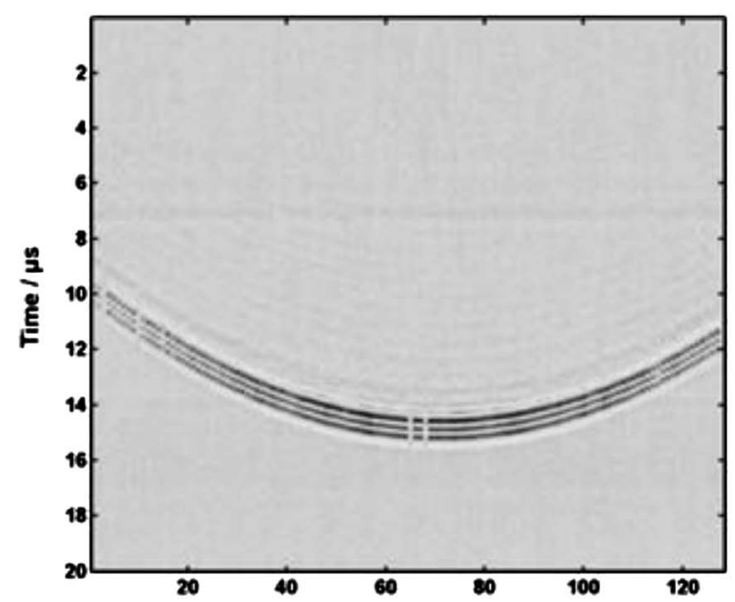

(a)

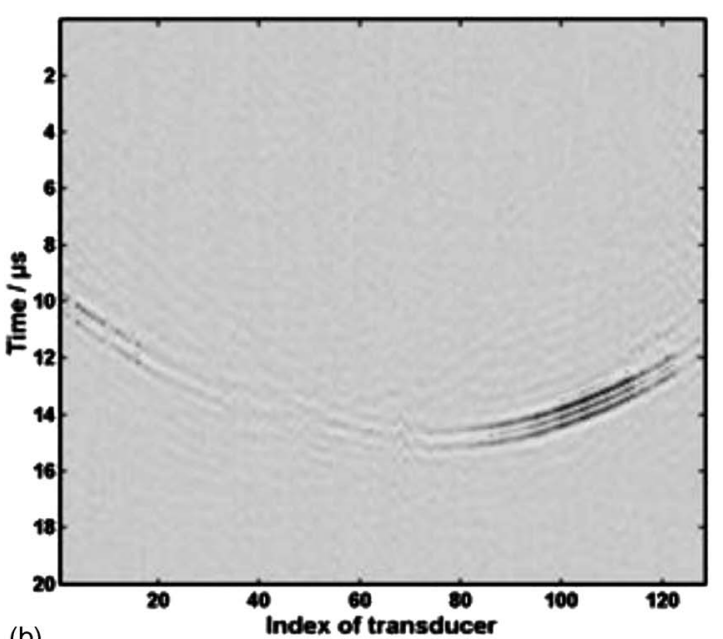

(b)

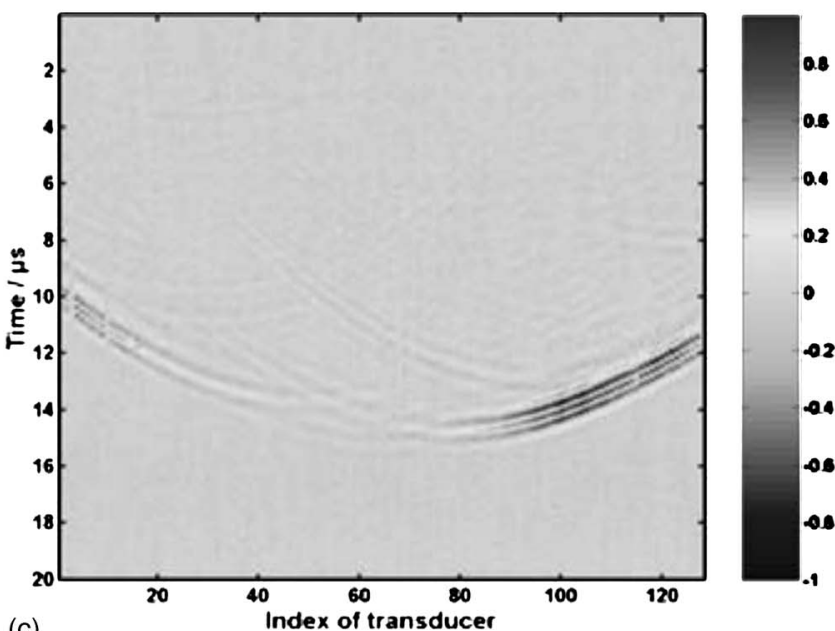

(c)

FIG. 6. Typical emissions: (a) A—obtained with a time-reversal experiment in water, (b) B-obtained with a time-reversal experiment through the ribs, and (c) $\mathrm{C}$-obtained by projection of emission A orthogonally to the first 40 eigenvectors.

$$
\mathbf{A}_{\text {projected }}(\boldsymbol{\omega})=\mathbf{A}(\boldsymbol{\omega})-\sum_{i=1}^{i_{\max }}\left(\mathbf{V}_{i}^{\dagger}(\boldsymbol{\omega}) \mathbf{A}(\boldsymbol{\omega})\right) \mathbf{V}_{\mathbf{i}}(\boldsymbol{\omega}),
$$

where $\boldsymbol{V}_{i}$ is the $i$ th eigenvector and $i_{\max }$ is the number of eigenvectors belonging to the first set of eigenvectors. An
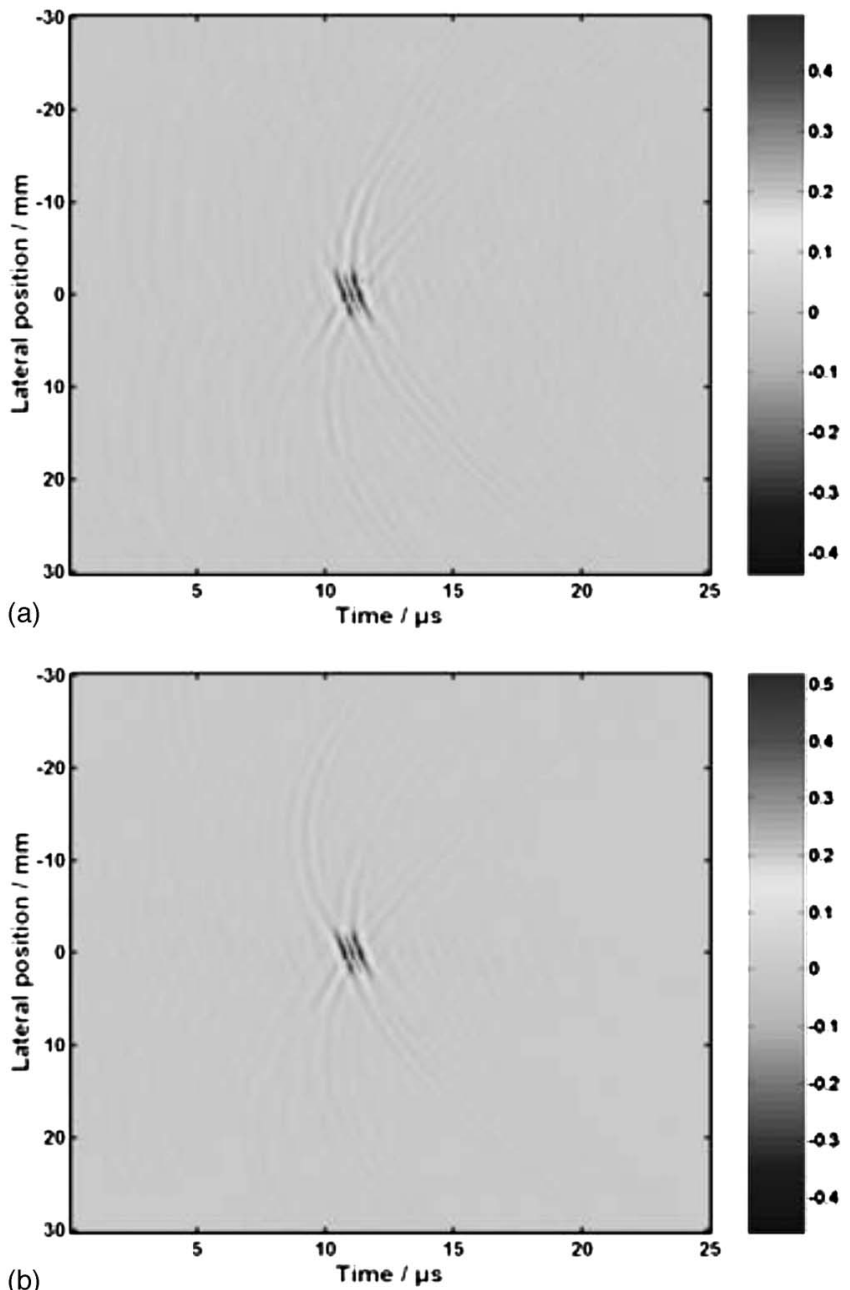

(b)

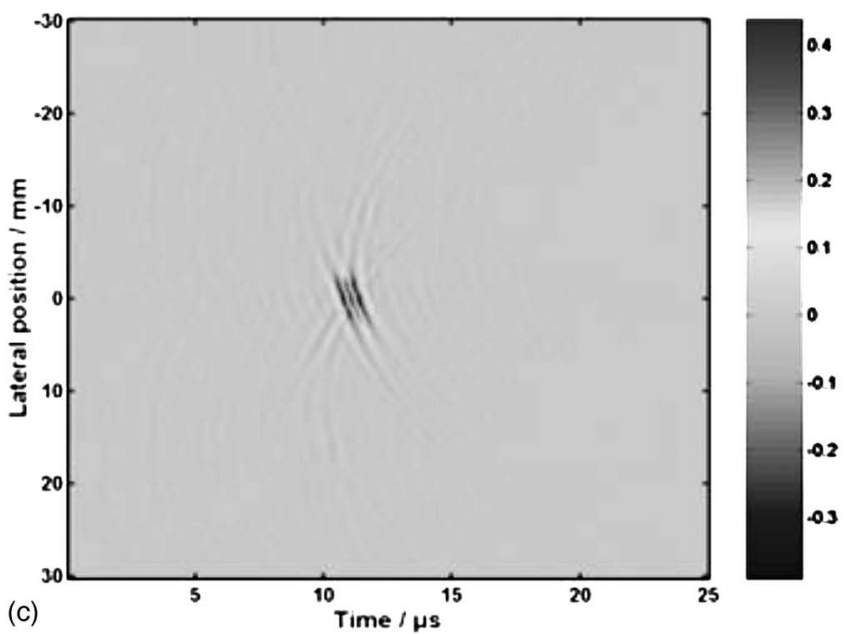

FIG. 7. Pressure field measured in the focal plane after (a) emission A, (b) emission $\mathrm{B}$, and (c) emission $\mathrm{C}$.

inverse Fourier transform is then performed to obtain a temporal signal.

\section{II.F. Experimental protocol}

In order to demonstrate the efficiency of the projection method, three types of focusing emissions have been compared: 


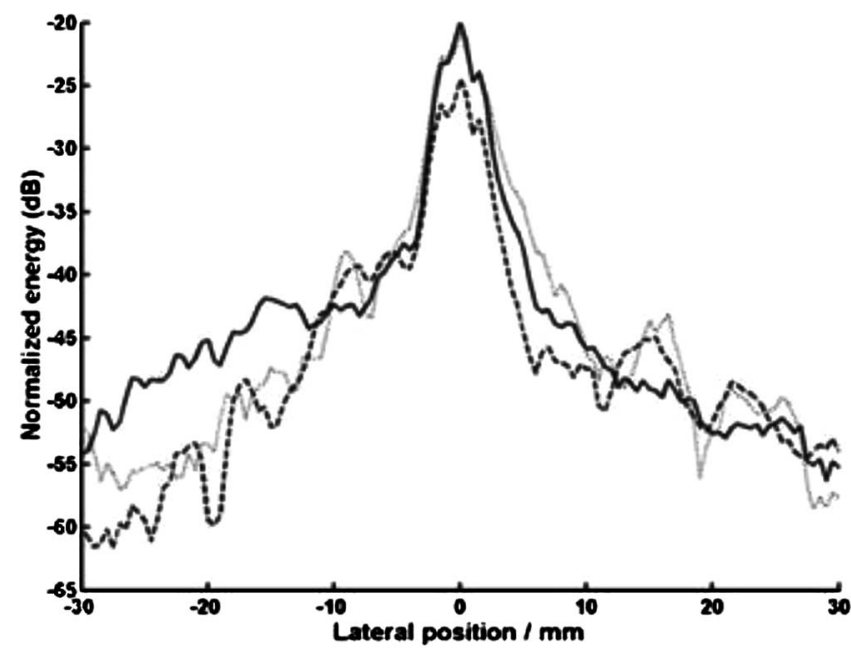

FIG. 8. Normalized energy distribution ( $\mathrm{dB}$ scale) in the focal plane obtained with emission A (dashed line), B (full line), and C (dotted line). The lateral position at $0 \mathrm{~mm}$ corresponds to the center of the focal spot.

(1) Without the ribs (free space), the impulse response from the desired focal spot to the array was recorded and time reversed, providing emission A. It is well known that emission A focuses in free space at the desired focal spot.

(2) The same measurement was done through the ribs, providing emission $\mathrm{B}$.

(3) Finally, a DORT experiment was run, and the eigenvector sets were discriminated as described in Sec. II D. To allocate each eigenvector to the correct subset, and find $i_{\max }$, the singular values distribution must be examined.

A typical distribution of singular values is displayed in Fig. 3 for each of the five sequences. $i_{\max }$ was set to 40 : Beyond that value, the singular values can be neglected. The numerical backpropagations of the eigenvectors at the central frequency showed that beyond this value, the eigenvectors did not focus on specific points on the ribs but would rather spread energy over a large number of points (see Fig. 4). On the other hand, Fig. 5 shows that the first eigenvectors focus where the ribs are. The same value (40) was kept for all frequencies. Emission A was then projected orthogonally to the first set of eigenvectors, providing emission $\mathrm{C}$.

Finally, the focal spot obtained for these three emissions through the ribs and the field obtained in the plane of the ribs after they were removed were measured with a $0.5 \mathrm{~mm}$ pitch. This sequence was repeated five times for five different geometrical configurations. Only the lateral position of the ribs changed from a configuration to the other: The center of the central rib varied from -10 to $0 \mathrm{~mm}$ and the intercostal space from 12 to $17 \mathrm{~mm}$.

\section{RESULTS}

Typical emissions A-C obtained for the third set of measures are shown in Fig. 6. The corresponding pressure fields measured in the focal plane along the $x$ axis are displayed in Fig. 7 as a function of time. The square of the corresponding
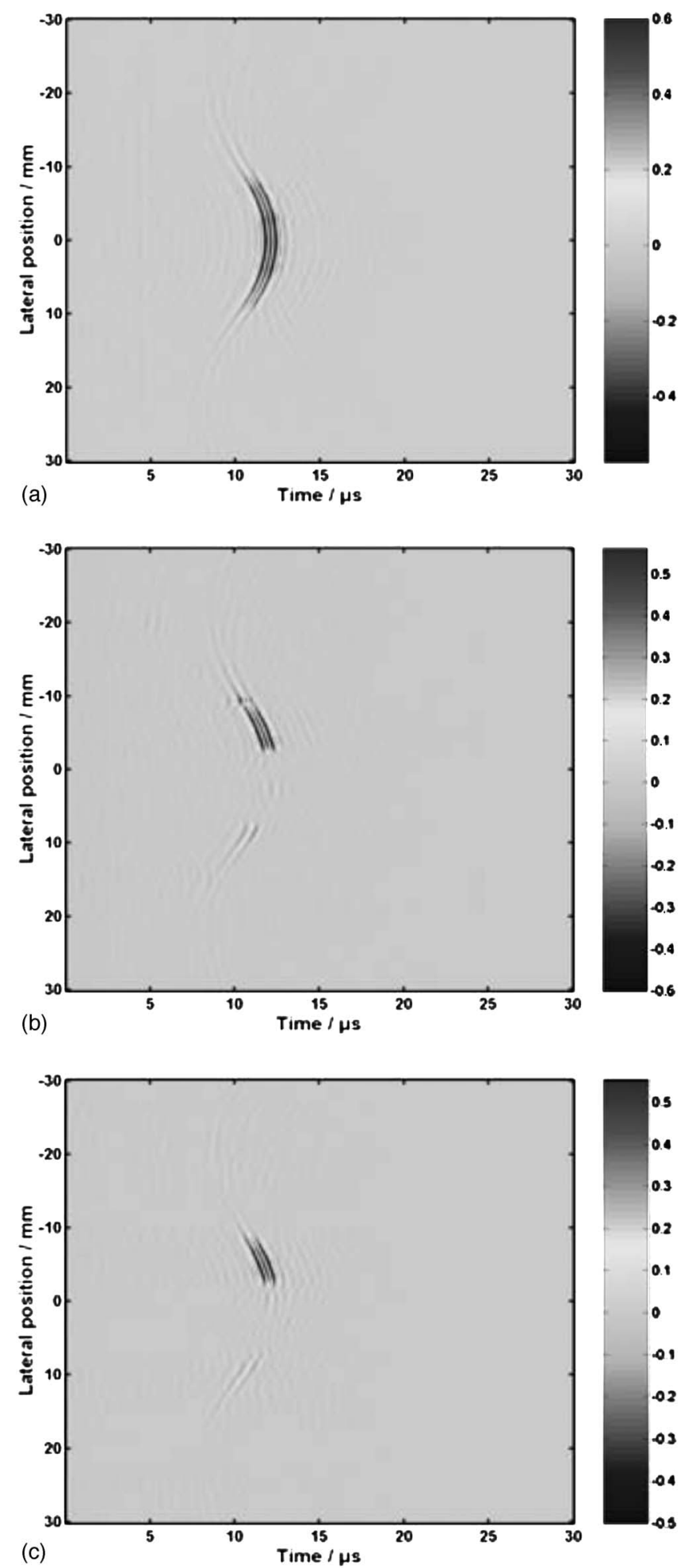

FIG. 9. Pressure field measured in the plane of the ribs after (a) emission A, (b) emission $\mathrm{B}$, and (c) emission $\mathrm{C}$. The lateral position at $0 \mathrm{~mm}$ corresponds to the center of the focal spot.

time peak amplitude is plotted in a dB scale in Fig. 8. In order to estimate the efficiency of the focusing, each plot is normalized by the total emitted energy (sum over the transducers of the square of the time peak of the emitted B-scan).

The three beam patterns are very similar with the same 
TABLE I. Quality of focusing.

\begin{tabular}{|c|c|c|c|c|c|c|c|c|c|}
\hline \multirow[b]{2}{*}{$\begin{array}{c}\text { Index of } \\
\text { the } \\
\text { experiment }\end{array}$} & \multicolumn{3}{|c|}{$\begin{array}{c}\text { Emission } \mathrm{A} \\
\text { (time reversal in water) }\end{array}$} & \multicolumn{3}{|c|}{$\begin{array}{c}\text { Emission B } \\
\text { (time reversal with the ribs) }\end{array}$} & \multicolumn{3}{|c|}{$\begin{array}{c}\text { Emission } \mathrm{C} \\
\text { (projection of emission } \mathrm{A} \text { ) }\end{array}$} \\
\hline & $\begin{array}{l}-3 \mathrm{~dB} \\
\text { width } \\
(\mathrm{mm})\end{array}$ & $\begin{array}{l}-6 \mathrm{~dB} \\
\text { width } \\
(\mathrm{mm})\end{array}$ & $\begin{array}{c}-12 \mathrm{~dB} \\
\text { width } \\
(\mathrm{mm})\end{array}$ & $\begin{array}{c}-3 \mathrm{~dB} \\
\text { width } \\
(\mathrm{mm})\end{array}$ & $\begin{array}{c}-6 \mathrm{~dB} \\
\text { width } \\
(\mathrm{mm})\end{array}$ & $\begin{array}{c}-12 \mathrm{~dB} \\
\text { width } \\
(\mathrm{mm})\end{array}$ & $\begin{array}{c}-3 \mathrm{~dB} \\
\text { width } \\
(\mathrm{mm})\end{array}$ & $\begin{array}{c}-6 \mathrm{~dB} \\
\text { width } \\
(\mathrm{mm})\end{array}$ & $\begin{array}{c}-12 \mathrm{~dB} \\
\text { width } \\
(\mathrm{mm})\end{array}$ \\
\hline 1 & 1 & 4 & 6.5 & 1 & 4 & 5 & 1 & 4 & 6.5 \\
\hline 2 & 2 & 4 & 5.5 & 1 & 3 & 5 & 1 & 4 & 5.5 \\
\hline 3 & 2 & 4 & 6 & 1 & 4 & 5 & 2 & 4 & 6.5 \\
\hline 4 & 3 & 5 & 10 & 3 & 4.5 & 6 & 3.5 & 5 & 8 \\
\hline 5 & 3 & 4.5 & 7 & 2.5 & 4 & 6.5 & 3 & 4.5 & 7 \\
\hline
\end{tabular}

$-6 \mathrm{~dB}$ width. One has to note that depending on the experiment, the $-6 \mathrm{~dB}$ width ranged from 3 to $5 \mathrm{~mm}$ (Table I), which is a small enough range for therapeutic purposes. ${ }^{15}$ Emission $\mathrm{A}$ is, on average, $5 \mathrm{~dB}$ lower than emissions $\mathrm{B}$ and $\mathrm{C}$ because part of the energy is absorbed or scattered by the ribs.

On the opposite hand, in the plane of the ribs, the fields differ significantly, as can be seen in Fig. 9 (obtained with the third set of measures). For both emissions B and C [Fig. $10(\mathrm{a})]$, the amplitude of the field drops significantly between -3 and $+10 \mathrm{~mm}$. This corresponds to the position of the central rib. A closer look at the same plot displayed in a $\mathrm{dB}$ scale [Fig. 10(b)] shows that the amplitude drop is not constant over the central rib: It decreases by $12-28 \mathrm{~dB}$. The SAR gains were calculated for each set of measures for all three emissions. The results are gathered in Tables II and III. Experiments are ordered with increasing intercostal distance.

For emission A, the mean SAR gain is smaller than 1: The ribs heat more than the region to treat, leading to unavoidable skin burns. For emissions B and C, the mean SAR gain is always larger than 1 due to ribs sparing. Not surprisingly, the SAR gain is always larger with the invasive approach (emission B).

\section{DISCUSSION}

For transcribes liver therapy the set of emission signals must meet two conditions: They must focus at the chosen location and avoid energy deposition on the highly absorbing ribs. The first condition was seen to be easily achievable because the abreacting effect of the ribs is small. In fact, the reflection and absorption by the ribs are so high that the amplitude of the field that propagated through the ribs (and has been distorted by the ribs) is negligible compared to that of the field that propagated in the intercostal space. The location of the focal spot has been shown to be correct and its width is acceptable for liver ablation.

The second condition is harder to achieve. Even if very little energy is sent onto the ribs, the SAR gain may be small because the absorption coefficient of the bone is high. This is indeed what was observed. Even though emissions B and C are very similar (and so are the resulting fields measured on the ribs' surface), their SAR gains differ by a slightly higher pressure field on the ribs with emission C. As a result, the SAR gain is smaller with the noninvasive approach.

Nevertheless, the SAR gain is significantly enhanced by the projection technique. It is 20- to 106-fold higher compared to the noncorrected approach, as shown in Table II. Variations in the SAR gain are observed from one set of measures to another for each type of emission. For emission A, the variations can be explained by a simple geometric factor: The larger the rib surface, on the journey between the transducers array and the focal spot, the smaller the SAR gain will be. In cases 1 and 2, the intercostal space is bigger than that in case 3, which is bigger than that in case 5. So, the SAR gain decreases because more energy is sent onto the ribs. In case 4 one of the gaps between the two ribs was placed just in front of the middle of the transducers array,

TABLE II. Comparison of the SAR gain obtained with three different emissions in five different geometries (calculated by taking the average on the central rib). The last column gives the enhancement of the SAR gain for emission $\mathrm{C}$.

\begin{tabular}{cccccc}
\hline \hline $\begin{array}{c}\text { Index of } \\
\text { the } \\
\text { experiment }\end{array}$ & $\begin{array}{c}\text { Intercostal } \\
\text { spacing } \\
(\mathrm{mm})\end{array}$ & $\begin{array}{c}\text { Average SAR gain } \\
\text { for emission A (times } \\
\text { reversal in water) }\end{array}$ & $\begin{array}{c}\text { Average SAR gain for } \\
\text { emission B (time } \\
\text { reversal with the ribs) }\end{array}$ & $\begin{array}{c}\text { Average SAR gain } \\
\text { for emission C } \\
\text { (projection of } \\
\text { emission A) }\end{array}$ & $\begin{array}{c}\text { SAR gain(C) } \\
\text { SAR gain(A) }\end{array}$ \\
\hline 1 & 30 & 0.12 & 13 & 3.0 & 25 \\
2 & 29 & 0.12 & 23 & 7.5 & 62.5 \\
3 & 24 & 0.085 & 11 & 9.0 & 106 \\
4 & 24 & 0.14 & 7.0 & 2.8 & 20 \\
5 & 20 & 0.017 & 1.4 & 1.3 & 76 \\
\hline \hline
\end{tabular}



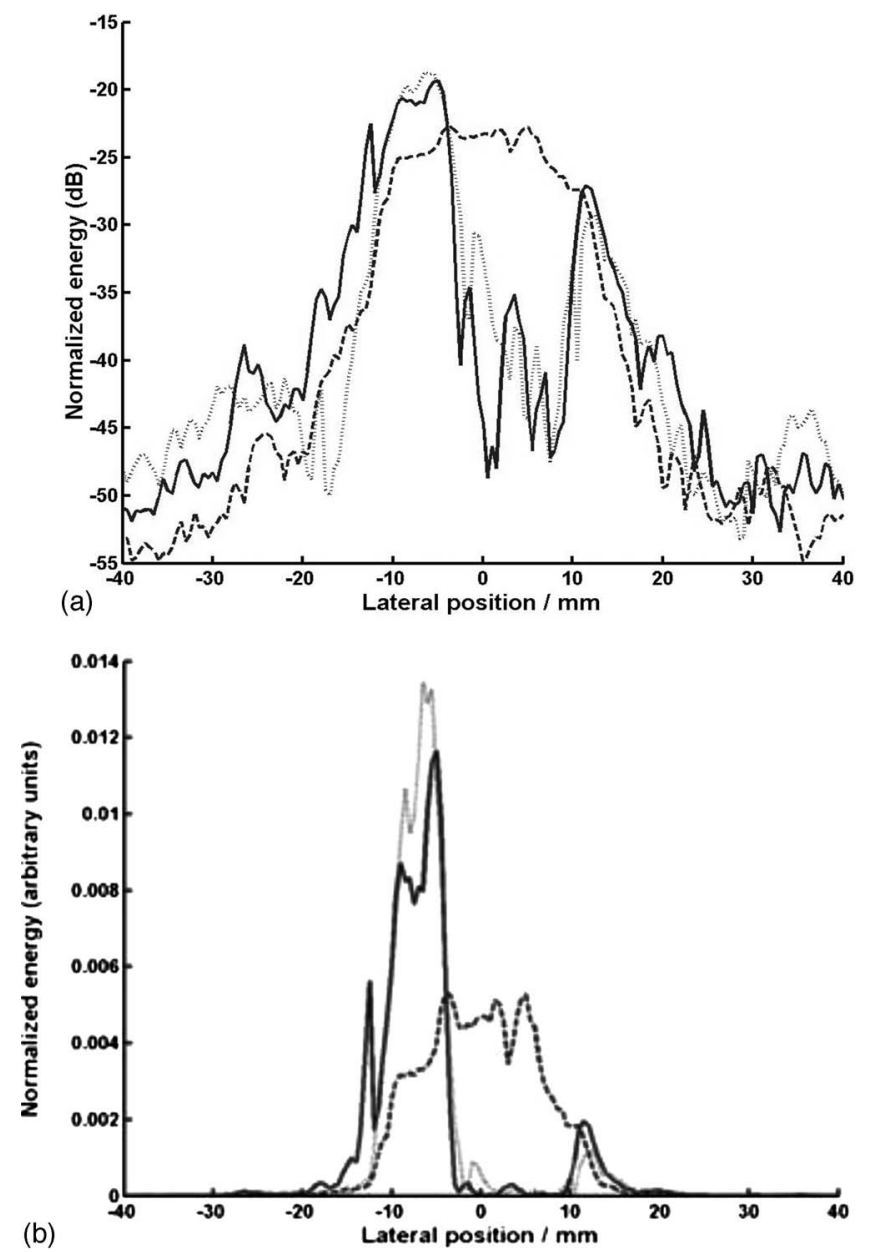

FIG. 10. Normalized energy distribution (left, dB scale; right, linear scale) in the plane of the ribs obtained with emission A (dashed line), B (solid line), and $\mathrm{C}$ (dotted line). The lateral position at $0 \mathrm{~mm}$ corresponds to the center of the focal spot.

where the energy is concentrated. The surrounding ribs therefore only saw the weaker lateral parts of the emission, thus increasing the SAR gain.

The same applies to emission B, if the intercostal space is smaller, less energy can be sent to the focal spot, leading to an expected decrease in SAR gain from one experiment to another. Nevertheless, the time-reversal approach is more complex and in some cases, depending on the geometry, en- ergy might be deposited on the ribs. This would mean that some energy has crossed the ribs on its way to the array in the first step of the time-reversal process. As some ribs were thinner on their sides, ultrasound rays arriving on these points could be incompletely absorbed. Furthermore, guided modes at the surface of the ribs can also radiate toward the array in the first step of the time-reversal process. Of course, the amount of transmitted energy varies depending on the exact geometry (namely, the angle between the rib and the transducer array). Even though no obvious trend can be related to intercostal spacing, the SAR gain is strongly enhanced by this time-reversal approach in all cases, confirming the results of Aubry et al. ${ }^{17}$

Similarly, the efficiency of the noninvasive DORT projection approach is not constant. As for the time-reversal approach, different amounts of information can be collected with the DORT method depending on the exact geometry. In this case, SAR gain seems to depend less on the intercostal space than on the orientation of the ribs. Nevertheless, the efficiency of this novel noninvasive approach is clearly demonstrated by the finding that the SAR gain is at least 20 -fold better than without correction.

Another approach for noninvasive transrib focusing consists of placing a set of virtual sources in the intercostal spaces in order to force the beam to go through these sources without sonicating on the ribs. Botros et al. proposed using a so-called "hybrid virtual arrays" made of such sources. ${ }^{15}$ To do so, they proposed to solve optimal synthesis of complex pressure in the virtual array plane while minimizing power deposition at critical points. According to Tables II and III, the results obtained by the method introduced in this article are similar to those obtained numerically by Botros et al. Nevertheless, the DORT method does not require imaging of the ribs and placement of virtual sources in between the expected location of the ribs. Moreover, the hybrid virtual array method requires computing the pseudoinverse transfer matrices between the virtual arrays and the transducers on one hand and between the virtual arrays and the focal plane on the other hand, making it difficult to perform in real time for a therapeutic 2D array.

\section{CONCLUSION}

In this study, the DORT method was applied to chest wall models composed of three ribs immerged in water. Contrary

TABLE III. Comparison of the SAR gain obtained with three different emissions in five different geometries (calculated by taking the maximum value on the central rib). The last column gives the enhancement of the SAR gain for emission $\mathrm{C}$.

\begin{tabular}{cccccc}
\hline \hline $\begin{array}{c}\text { Index of } \\
\text { the } \\
\text { experiment }\end{array}$ & $\begin{array}{c}\text { Intercostal } \\
\text { spacing } \\
(\mathrm{mm})\end{array}$ & $\begin{array}{c}\text { Lowest SAR gain } \\
\text { for emisson A (times } \\
\text { reversal in water) }\end{array}$ & $\begin{array}{c}\text { Lowest SAR gain } \\
\text { for emission B (time } \\
\text { reversal with the ribs) }\end{array}$ & $\begin{array}{c}\text { Lowest SAR gain } \\
\text { for emission C } \\
\text { (projection of } \\
\text { emission A) }\end{array}$ & $\begin{array}{c}\text { SAR gain(C) } \\
\text { SAR gain(A) }\end{array}$ \\
\hline 1 & 30 & 0.11 & 3.2 & 1.0 & 9.6 \\
2 & 29 & 0.094 & 4.2 & 1.7 & 18 \\
3 & 24 & 0.067 & 1.7 & 1.1 & 16 \\
4 & 24 & 0.054 & 1.1 & 0.58 & 10 \\
5 & 20 & 0.0136 & 0.4 & 0.33 & 24 \\
\hline \hline
\end{tabular}


to most DORT studies, the information provided by the measured array response matrix was used to avoid sending energy on to the scatterers instead of focusing on them. Experimental results showed that the projection of an emission orthogonally to the eigenvectors associated with the scatterers leads to an emission that avoids these scatterers. The projected emission was compared to the original and to an invasive time-reversal experiment. It was shown that the projected emission leads to a focusing similar to the timereversed emission and could enhance the efficacy of the ribs sparing by a factor of 100 .

Such a noninvasive approach could be implemented clinically in order to avoid skin burns in transrib liver therapy. The method could be automated because all the information is directly contained in the singular vectors. Little computations would be needed (on the order of the number of transducers used). The use of multielement therapeutic arrays would be mandatory but such systems are becoming more and more widespread. ${ }^{26-30}$

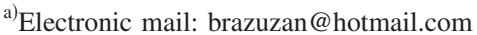

${ }^{1}$ A. Gelet, J. Y. Chapelon, J. Margonari, Y. Theilliere, F. Gorry, and R. Souchon, "High intensity focused ultrasound experimentation on human benign prostatic hypertrophy," Eur. Urol. 23, 44-47 (1993).

${ }^{2}$ S. Madersbacher, C. Kratzik, M. Susani, and M. Marberger, "Tissue ablation in benign prostatic hyperplasia with high intensity focused ultrasound," J. Urol. (Paris) 152, 1956-1961 (1994).

${ }^{3}$ S. Madersbacher, M. Pedevilla, L. Vingers, M. Susani, and M. Marberger, "Effect of high intensity focused ultrasound on human prostate cancer in vivo," Cancer Res. 55, 3346-3351 (1995).

${ }^{4}$ N. T. Sanghvi, F. J. Fry, R. Bihrle, R. S. Foster, M. H. Phillips, J. Syrus, A. V. Zaitsev, and C. W. Hennige, "Noninvasive surgery of prostate tissue by high-intensity focused ultrasound," IEEE Trans. Ultrason. Ferroelectr. Freq. Control 43, 1099-1110 (1996).

${ }^{5}$ A. Gelet, J. Y. Chapelon, R. Bouvier, R. Souchon, C. Pangaud, A. F. Abdelrahim, D. Cathignol, and J. M. Dubernard, "Treatment of prostate cancer with transrectal focused ultrasound: Early clinical experience," Eur. Urol. 29, 174-183 (1996).

${ }^{6}$ F. M. Fennessy and C. M. Tempany, "A review of magnetic resonance imaging-guided focused ultrasound surgery of uterine fibroids," Top Magn. Reson Imaging 17(3), 173-179 (2006).

${ }^{7}$ G. Vallancien, M. Harouni, and B. Veillon, "Focused extracorporeal pyrotherapy: Feasibility study in man,” J. Endourol 6, 173-180 (1992).

${ }^{8}$ J. E. Kennedy, F. Wu, and G. R. ter Haar, "High intensity focused ultrasound for the treatment of liver tumours," Ultrasonics 42, 931-935 (2004).

${ }^{9}$ J. E. Kennedy, R. L. Clarke, and G. R. ter Haar, "The effects of absorbers such as ribs in the HIFU Beam-path on the focal profile," Proceedings of the Second International Symposium on Therapeutic Ultrasound (Center for Industrial \& Medical Ultrasound, Applied Physics Laboratory, University of Washington, Seattle, 2002), pp. 185-192.

${ }^{10} \mathrm{~S}$. A. Goss, R. L. Johnston, and F. Dunn, "Comprehensive compilation of empirical ultrasonic properties of mammalian tissues II," J. Acoust. Soc.
}

Am. 68(1), 93-108 (1980).

${ }^{11}$ D. R. Daum, N. B. Smith, R. King, and K. Hynynen, "In vivo demonstration of noninvasive thermal surgery of the liver and kidney using an ultrasonic phased array," Ultrasound Med. Biol. 25, 1087-1098 (1999).

${ }^{12} \mathrm{~F}$. Wu, Z. B. Wang, and W. Z. Chen, "Extracorporeal high intensity focused ultrasound ablation in the treatment of 1038 patients with solid carcinomas in China: An overview," Ultrason. Sonochem. 11(3-4), 149154 (2004).

${ }^{13}$ J. J. Li, G. L. Xu, and M. F. Gu, "Complications of high intensity focused ultrasound in patients with recurrent and metastatic abdominal tumors," Cognit. Sci. Q. 13(19), 2747-2751 (2007).

${ }^{14} \mathrm{~F}$. Wu et al., "Extracorporeal high intensity focused ultrasound ablation in the treatment of patients with large hepatocellular carcinoma," Ann. Surg. Oncol. 11, 1061-1069 (2004).

${ }^{15}$ Y. Y. Botros, E. S. Ebbini, and J. L. Volakis, "Two-step hybrid virtual array-ray (VAR) technique for focusing through the rib cage," IEEE Trans. Ultrason. Ferroelectr. Freq. Control 45, 989-1000 (1998).

${ }^{16} \mathrm{H}$. Liu et al., "Feasability of transrib focused ultrasound thermal ablation for liver tumors using a spherically curved 2D array: A numerical study," Med. Phys. 34, 3436-3448 (2007).

${ }^{17}$ J. F. Aubry, M. Pernot, F. Marquet, M. Tanter, and M. Fink, "Transcostal high intensity focused ultrasound: Ex vivo adaptative focusing feasibility study," Phys. Med. Biol. 53, 2937-2951 (2008).

${ }^{18}$ C. Prada and M. Fink, "Eigenmodes of the time-reversal operator: A solution to selective focusing in multiple target media," Wave Motion 20, 151-163 (1994)

${ }^{19}$ H. C. Song, S. Kim, W. S. Hodgkiss, and W. A. Kuperman, "Environmentally adaptive reverberation nulling using a time reversal mirror," J. Acoust. Soc. Am. 116, 762-768 (2004).

${ }^{20} \mathrm{~F}$. J. Fry and J. E. Barger, "Acoustical properties of the human skull," J. Acoust. Soc. Am. 63, 1576-1590 (1978)

${ }^{21} \mathrm{~F}$. Wu, J. L. Thomas, and M. Fink, "Time reversal of ultrasonic field. Part II. experimental results," IEEE Trans. Ultrason. Ferroelectr. Freq. Control 39(5), 567-578 (1992).

${ }^{22}$ M. Pernot, J.-F. Aubry, M. Tanter, A.-L. Boch, F. Marquet, M. Kujas, D. Seilhean, and M. Fink, "In vivo transcranial brain surgery with an ultrasonic time reversal mirror," J. Neurosurg. 106(6), 1061-1066 (2007).

${ }^{23} \mathrm{C}$. Prada, "Detection and imaging in complex media with the DORT method," Top. Appl. Phys. 84, 107-133 (2002).

${ }^{24}$ A. Aubry, J. de Rosny, J. G. Minonzio, C. Prada, and M. Fink, “Gaussian beams and Legendre polynomials as invariants of the time-reversal operator for a large rigid cylinder," Izv. Akad. Nauk Arm. SSR, Fiz. 120, 2746-2754 (2006).

${ }^{25} \mathrm{D}$. H. Chambers, "Target characterization using time-reversal symmetry of wave propagation," Int. J. Mod. Phys. B 21(20), 3511-3555 (2007).

${ }^{26}$ E. S. Ebbini, H. Yao, and A. Shrestha, "Dual-mode ultrasound phased arrays for image-guided surgery," Ultrason. Imaging 28(2), 65-82 (2006).

${ }^{27}$ M. Tanter, M. Pernot, J. F. Aubry, G. Montaldo, F. Marquet, and M. Fink, "Compensating for bone interfaces and respiratory motion in high intensity focused ultrasound," Int. J. Hyperthermia 23(2), 141-151 (2007).

${ }^{28}$ J. F. Aubry et al., "Ultrasonic arrays: New therapeutic developments," J. Radiol. 88(11), 1801-1809 (2007).

${ }^{29} \mathrm{~K}$. Hynynen, "Ultrasound for drug and gene delivery to the brain," Adv. Drug Delivery Rev. 60(10), 1209-1217 (2008).

${ }^{30}$ F. A. Jolesz and N. McDannold, "Current status and future potential of MRI-guided focused ultrasound surgery," J. Magn. Reson Imaging 27(2), 391-399 (2008). 